\title{
Teleconsultations and Shared Care in Pediatric Oncology During COVID-19
}

\author{
Shuvadeep Ganguly ${ }^{1} \cdot$ Sameer Bakhshi ${ }^{1}$
}

Received: 21 August 2020 / Accepted: 10 September 2020 / Published online: 22 September 2020

(C) Dr. K C Chaudhuri Foundation 2020

The recent coronavirus disease-19 pandemic (COVID-19) presented a significant challenge in the effective care delivery for children with cancer across the globe. The data on clinical outcome of COVID-19 in childhood cancer patients is limited with very few cases severe enough to require intensive care [1]. Still, keeping in mind the immunosuppressive nature of anti-neoplastic therapy and our evolving understanding of the disease, pediatric oncology services have to tread carefully between treating cancer and limiting COVID-19 in this vulnerable population.

With healthcare centres throughout the world grappling with COVID-19 pandemic with resource optimization, all aspects of cancer treatment were adversely affected, including ancillary services like blood product availability [2]. Drawing on the early experience in Italy, a consensus based guideline was formulated by International Childhood Cancer Organizations to adapt treatment strategy of childhood cancer patients keeping in mind resource limitations during the pandemic [3].

Our centre is a government tertiary care healthcare institution, which was also concurrently designated as a COVID-19 hospital during this pandemic. This led to manpower diversion thereby curtailing all aspects of non-COVID-19 healthcare services. India also simultaneously instituted lockdown measures early in the pandemic which led to further difficulty for parents/caregivers to access timely care. Our team formulated a multi-pronged strategy to adapt our treatment services considering these local challenges [4].

Initial triaging of patients and deciding intent of treatment was the cornerstone of the strategy. For children with palliative intent, hospital visits and intravenous chemotherapy were

Sameer Bakhshi

sambakh@hotmail.com

1 Department of Medical Oncology, Dr. B.R.A. Institute Rotary Cancer Hospital, All India Institute of Medical Sciences, New Delhi 110029, India deferred as much as possible and oral metronomic therapy was administered wherever feasible [5]. We also adapted our chemotherapy protocols for various solid malignancies utilizing oral therapy and deferring hospital visits as a temporary measure especially for families living far away from hospital [6]. On the other hand, for aggressive malignancies like acute leukemia or Non-Hodgkin lymphoma (NHL) with curative intent, all efforts were made to institute or continue therapy according to protocol without any delay. Proper multidisciplinary co-ordination was maintained and life-saving surgeries with curative intent for disease like bone sarcoma were carried out with all proper precautions during this pandemic period [7].

We kept a significantly low threshold for testing any symptomatic patient for severe acute respiratory syndrome coronavirus 2 (SARS-CoV-2) and isolate any positive cases at the earliest. Over the course of last four months of this pandemic (April to July 2020), even with curtailing of healthcare services at our centre, 80 new cases (age $\leq 21$ years) were registered and 39 children with acute leukemia and lymphoma were started on therapy. Among the children under our follow-up, 12 children turned positive for SARS-CoV-2 with fatal outcome in two (16.6\%) of them; both these cases were having relapse/refractory cancer. Unfortunately, another one of our patient committed suicide on fear of contracting COVID-19 which underlines the excess of fear, stress, and stigma this disease has caused among common populace.

During this pandemic situation, the Government of India devised policies to strengthen telemedicine services and encouraged the adoption of digital healthcare [8]. Taking cue from the same, we contacted families/caregivers of a significant subset of routine outpatient oncology patients telephonically and also issued prescription via e-mail facility. Support were provided to create e-mail facilities for families who were not well versed with technology by involving alternate family members. All childhood cancer survivors were followed up telephonically, advised to undergo investigations as per protocol locally, the reports of which were then evaluated via e- 
mail facility. This strategy of teleconsultation was also predominantly utilized for patients under non-intensive care like children with acute lymphoblastic leukemia/Langerhans cell histiocytosis under maintenance therapy or patients on comparatively simpler protocols like Hodgkin lymphoma. Chemotherapy plans were decided after reviewing reports, and prescriptions were shared via e-mail. Families were then advised to undergo chemotherapy at local healthcare institutions with pediatric facilities even including intrathecal drug administrations. During these last 4 months, our team and patients' families readily adopted telemedicine services with number of teleconsultations rising from 32 in month of April 2020 to 197 in month of July 2020. Although there was a significant decrease in number of routine outpatient consultations (39\% as compared to similar period last year), total 313 children were managed on teleconsultation basis, which constitutes a sizeable $15.5 \%$ of cumulative outpatient visits during this period.

Childhood cancer services in India is predominantly concentrated in few tertiary care institutes in first tier cities leading to inequitable and often delayed care [9]. The extra-ordinary situation posed by this pandemic forced us to develop collaborative model to ensure continuity of care by involving local pediatric healthcare facilities, akin to the "shared care model" as practised in some Western countries [10].

Being a tertiary care institution located at national capital, majority of families accessing our centre belongs to far away places [6]. Staying near the hospital during the whole treatment course and travelling regularly from their residential states for physical consultations during maintenance/followup period adds a significant indirect financial burden to an already prohibitive cost of cancer care for most of the families. Although cost effectiveness was not formally analysed, we believe utilization of teleconsultation along with shared care model, wherever feasible, will go a long away in easing cost of care and reduce this burden of regular travel. During this pandemic period, we observed that majority of parents/caregivers were comfortable using e-mail for teleconsultations and were willing to continue the same even when the crisis abates. This is exemplified by the fact that 42 families ( $13.4 \%$ of all teleconsultations) sought re-consultation via e-mail rather than a physical visit even though travel restrictions have been eased currently. However, certain families faced problems procuring chemotherapeutic drugs at their local places of residence for which help, and logistic support were provided with help of philanthropic organizations. Confidence of parents on local healthcare facilities is another concern which needs time to develop and evolve.

Our experience with telemedicine and developing this "shared care" model suggests that it is a feasible approach, and that even after COVID-19 pandemic wanes away, this will perhaps remain as a healthy legacy of the pandemic.
Acknowledgements We sincerely acknowledge the dedicated efforts of entire pediatric oncology team of our centre especially nursing staff, dietician, data management professional and all supporting staff in formulating strategy and managing children with cancer during this exceptional pandemic situation.

Authors' Contributions SB and SG conducted review, data interpretation and wrote the manuscript. SB will act as guarantor for this paper.

\section{Compliance with Ethical Standards}

Conflict of Interest None.

\section{References}

1. Boulad F, Kamboj M, Bouvier N, Mauguen A, Kung AL. COVID19 in children with cancer in New York city. JAMA Oncol. 2020. Available at: https://jamanetwork.com/journals/jamaoncology/ fullarticle/2766112. Accessed 8 Aug 2020.

2. Saab R, Obeid A, Gachi F, et al. Impact of the coronavirus disease 2019 (COVID-19) pandemic on pediatric oncology care in the Middle East, North Africa, and West Asia Region: a report from the Pediatric Oncology East and Mediterranean (POEM) Group. Cancer. 2020. https://doi.org/10.1002/cncr.33075.

3. Sullivan M, Bouffet E, Rodriguez-Galindo C, et al. The COVID-19 pandemic: a rapid global response for children with cancer from SIOP, COG, SIOP-E, SIOP-PODC, IPSO, PROS, CCI, and St Jude Global. Pediatr Blood Cancer. 2020. Available at: https:// onlinelibrary.wiley.com/doi/abs/10.1002/pbc.28409. Accessed 8 Aug 2020.

4. Garg V, Bakhshi S, Gupta G, Pushpam D. Managing pediatric cancer patients in COVID19 pandemic. Indian J Pediatr. 2020;87:558.

5. Pramanik R, Bakhshi S. Metronomic therapy in pediatric oncology: a snapshot. Pediatr Blood Cancer. 2019. Available at: https:// onlinelibrary.wiley.com/doi/abs/10.1002/pbc.27811. Accessed 8 Aug 2020.

6. Pushpam D, Bakhshi S, Agarwala S. Chemotherapy adaptations in a referral tertiary care center in India for ongoing therapy of pediatric patients with solid tumors during COVID19 pandemic and lockdown. Pediatr Blood Cancer. 2020. Available at: https:// onlinelibrary.wiley.com/doi/abs/10.1002/pbc.28428. Accessed 7 Aug 2020.

7. Kumar VS, Banjara R, Thapa S, et al. Bone sarcoma surgery in times of COVID-19 pandemic lockdown-early experience from a tertiary centre in India. J Surg Oncol. 2020. https://doi.org/10. $1002 /$ jso. 26112.

8. Telemedicine Practice Guidelines, Ministry of Health and Family Welfare, Government of India. Available at: https://www.mohfw. gov.in/pdf/Telemedicine.pdf. Accessed 8 Aug 2020.

9. Ganguly S, Kinsey S, Bakhshi S. Childhood cancer in India. Cancer Epidemiol. 2020;101679.

10. Kowalczyk JR, Samardakiewicz M, Pritchard-Jones K, et al. European survey on standards of care in paediatric oncology centres. Eur J Cancer. 2016;61:11-9.

Publisher's Note Springer Nature remains neutral with regard to jurisdictional claims in published maps and institutional affiliations. 